\title{
WHONDRS-GUI: a web application for global survey of surface water metabolites
}

\author{
Xinming Lin ${ }^{1}$, Huiying Ren ${ }^{1}$, Amy E. Goldman ${ }^{1}$, James C. Stegen ${ }^{\text {Corresp., } 1}{ }^{1}$, Timothy D. Scheibe ${ }^{1}$ \\ 1 Pacific Northwest National Laboratory, Richland, Washington, United States of America \\ Corresponding Author: James C. Stegen \\ Email address: James.Stegen@pnnl.gov
}

Background. The Worldwide Hydrobiogeochemistry Observation Network for Dynamic River Systems (WHONDRS) is a consortium that aims to understand complex hydrologic, biogeochemical, and microbial connections within river corridors experiencing perturbations such as dam operations, floods, and droughts. For one ongoing WHONDRS sampling campaign, surface water metabolite and microbiome samples are collected through a global survey to generate knowledge across diverse river corridors. Metabolomics analysis and a suite of geochemical analyses have been performed for collected samples through the Environmental Molecular Sciences Laboratory (EMSL). The obtained knowledge and data package inform mechanistic and data-driven models to enhance predictions of outcomes of hydrologic perturbations and watershed function, one of the most critical components in model-data integration. To support efforts of the multi-domain integration and make the ever-growing data package more accessible for researchers across the world, a Shiny/R Graphical User Interface (GUI) called WHONDRS-GUI was created.

Results. The Web application can be run on any modern Web browser without any programming or operational system requirements, thus providing an open, well-structured, discoverable dataset for WHONDRS. Together with a context-aware dynamic user interface, WHONDRS-GUI has functionality for searching, compiling, integrating, visualizing and exporting different data types that can easily be used by the community. The Web application and data package are available at https://data.ess-dive.lbl.gov/view/doi:10.15485/1484811, which enables users to simultaneously obtain access to the data and code and to subsequently run the web app locally. The WHONDRS-GUI is also available for online use at Shiny Server (https://xmlin.shinyapps.io/whondrs/). 
1 WHONDRS-GUI: A Web Application for Global Survey

2 of Surface Water Metabolites

3

4

5

6

7

8 Corresponding Author:

9 James C. Stegen ${ }^{1}$

10

11

12

13

14

15

16

17

18

19

20

21

22

23

24

25

26

27

28

29

30

31

32

33

34

35

36

37

38

39

\section{Abstract}

\section{Introduction}

Xinming Lin ${ }^{1}$, Huiying Ren ${ }^{1}$, Amy E. Goldman ${ }^{1}$, James C. Stegen ${ }^{1}$ and Timothy D. Scheibe ${ }^{1}$

${ }^{1}$ Pacific Northwest National Laboratory, Richland, WA, USA 99354

902 Battelle Blvd, Richland, WA 99354

Email address: James.Stegen@pnnl.gov

Background. The Worldwide Hydrobiogeochemistry Observation Network for Dynamic River Systems (WHONDRS) is a consortium that aims to understand complex hydrologic, biogeochemical, and microbial connections within river corridors experiencing perturbations such as dam operations, floods, and droughts. For one ongoing WHONDRS sampling campaign, surface water metabolite and microbiome samples are collected through a global survey to generate knowledge across diverse river corridors. Metabolomics analysis and a suite of geochemical analyses have been performed for collected samples through the Environmental Molecular Sciences Laboratory (EMSL). The obtained knowledge and data package inform mechanistic and data-driven models to enhance predictions of outcomes of hydrologic perturbations and watershed function, one of the most critical components in model-data integration. To support efforts of the multi-domain integration and make the ever-growing data package more accessible for researchers across the world, a Shiny/R Graphical User Interface (GUI) called WHONDRS-GUI was created. Results. The Web application can be run on any modern Web browser without any programming or operational system requirements, thus providing an open, well-structured, discoverable dataset for WHONDRS. Together with a context-aware dynamic user interface, WHONDRS-GUI has functionality for searching, compiling, integrating, visualizing and exporting different data types that can easily be used by the community. The Web application and data package are available at https://data.ess-dive.lbl.gov/view/doi:10.15485/1484811, which enables users to simultaneously obtain access to the data and code and to subsequently run the web app locally. The WHONDRSGUI is also available for online use at Shiny Server (https://xmlin.shinyapps.io/whondrs/).

Watershed systems play a critical role in human society and environmental health by providing ecosystem services such as drinking water for humans, water for irrigation and industrial activities, and habitats for plants and animals (Postel and Thompson Jr, 2005). The associated river corridors, including the river, adjacent land, and subsurface environments, are key components of watershed 
40

41

42

43

44

45

46

47

48

49

50

51

52

53

54

55

56

57

58

59

60

61

62

63

64

65

66

67

68

69

70

71

72

73

74

75

76

77

78

systems (Harvey and Gooseff, 2015). Recurring, episodic, or chronic perturbations to river corridors, such as those caused by dam operations, wastewater effluent, floods, storm pulses, and droughts, are common but difficult to study due to their transient nature. Understanding the connections among hydrologic, biogeochemical, and microbial function within river corridors is not only important to help predict the outcomes of these perturbations in terms of watershed function, but also to maintain a healthy environment (Graham, et al., 2019). The Worldwide Hydrobiogeochemistry Observation Network for Dynamic River Systems (WHONDRS) (Stegen and Goldman, 2018) is a global consortium of researchers and other interested parties dedicated to studying these connections.

WHONDRS generates knowledge across river corridors from local to global scales. For one ongoing study, surface water metabolite and microbiome samples are collected by local collaborators around the world through a global survey. This study aims to provide broad understanding of the functioning of dynamic river corridors. Metabolomics analysis via Fourier transform ion cyclotron resonance mass spectrometry (FTICR-MS) (Marshall, et al., 1998) and a supporting suite of geochemical analyses have been conducted through the Environmental Molecular Sciences Laboratory (EMSL) for the field-collected samples. The data obtained are intended to inform mechanistic and data-driven models to enhance predictions of the hydrobiogeochemical function of river corridors and watersheds, thus serving as a critical component of the model-data integration process (Scheibe, et al., 2018). However, as the number of collected samples increases, the resulting WHONDRS data package will be of size and complexity that makes it challenging for researchers to manage and search for the part of the data package that they are most interested in.

To support the efforts of multi-domain integration of physical, biological, and chemical processes within river corridors, a shiny-based web application called WHONDRS-GUI was created. This web app is intended to make the collected, ever-growing WHONDRS data package more accessible for researchers to use in performing new analyses or updating prior analyses, in keeping with the FAIR principles for scientific data management (Wilkinson, et al., 2016).

WHONDRS-GUI is a user-friendly graphical user interface (GUI) developed using R (R.Core.Team, 2018) and Shiny (Winston Chang, 2018). Like any other Shiny app, WHONDRSGUI can run on any modern Web browser without any programming or operational system requirements. It provides open, well-structured, discoverable data, visualization of specific data types, as well as a global map of all collected sampling sites. Together with a context-aware dynamic user interface, WHONDRS-GUI includes functionality for searching, compiling, integrating, and exporting different data types that can easily be used by the scientific community. The data types used to build WHONDRS-GUI include metadata of collected surface water samples, FTICR data and geochemistry data from analyses performed in EMSL, surface water hydrographs collected from existing instrumentation, and standardized photos of each field system (Stegen, et al., 2018). By engaging with the global scientific community, WHONDRS-GUI promotes sharing and extracting knowledge from the WHONDRS data package (Stegen, et al., 
2018), and enables researchers to better address science questions relevant to their individual sites as well as perform integrative cross-site analyses.

\section{Materials \& Methods}

\section{Data Sources and Description}

The data package used in this study is from an on-going global survey of surface water metabolite that started in 2018. Surface water metabolite and microbiome samples from streams and river corridors around the world were collected by local collaborators and sent to the EMSL for analysis. Metabolomics analysis via FTICR-MS and a suite of geochemical analyses were conducted on all samples at EMSL. The application is designed to take five different data types from the data package:

1. Metadata of collected surface water samples. Those are recorded by local collaborators who collected the surface water samples. The metadata include names of samplers, sample ID, sampling time, location, water temperature, distance from gauging station and river names of the water samples etc.

2. FTICR results. These are results of metabolomic analysis for collected surface water samples via FTICR-MS.

3. Basic geochemistry data. These are results of geochemistry analyses conducted for surface water samples.

4. Surface water hydrographs. These are open-access water-resources data of gauging stations downloaded from United States Geological Survey (USGS) website based on sampling locations of collected surface water samples.

5. Standardized photos taken from each field system. These are photos of field systems taken by collaborators.

Following data cleaning, manipulation and adjustment of raw data (performed using the 'dplyr' R package (Wickham, et al., 2015)), all available data types, except photos, were saved in commaseparated values (CSV) file format. Those processed data types were then used as inputs for building the WHONDRS-GUI. The WHONDRS-GUI provides the option of downloading all or part of the data package for users who are interested in performing their own analysis.

\section{Graphical User interface}

The WHONDRS-GUI interface was created in R console using Shiny and its supplementary packages. It is an interactive web application that can be run on a local machine using $\mathrm{R}$ or $\mathrm{R}$ studio. The web application is also deployed online by hosting to Shiny Server. This allows for fast access to as well as easy sharing of the web application through any modern Web browser.

The WHONDRS-GUI interface is organized into dashboard pages using the 'shinydashboard' package (Chang and Borges Ribeiro, 2017). It utilizes a tab-based dashboard format to make accessing each data type fast and intuitive. Data types, such as 'Metadata', 'FTICR', 'Geochemistry', 'Hydrograph' and 'Photo', are displayed with their names listed on the left-hand sidebar of the interface. The output tables, maps, and plots are displayed to the right of the sidebar, 
119 as the main body of the dashboard page. Users can explore each data type by clicking tab names

120

121

122

123

124

125

126

127

128

129

130

131

132

133

134

135

136

137

138

139

140

141

142

143

144

145

146

147

148

149

150

151

152

153

154

155

156

157

158

on the left. This will result in the corresponding output page appearing on the right.

The first tab on the left-hand sidebar of the interface, and the landing dashboard page when the web application is first loaded, is the 'Home' tab. Clicking this tab will lead users to the homepage of the web application. This page gives a short description of WHONDRS-GUI. Clicking the 'GET STARTED' action button below the description paragraph will lead users to the 'Help' dashboard page, where users can find more information about the web application and how to navigate it locally.

Following the 'Home' dashboard page are dashboards pages for the five available data types, including 'Metadata', 'FTICR', 'Geochemistry', 'Hydrograph' and 'Photo'. User input widgets on the top of these dashboard pages, allow users to search for a subset of data based on different options. As shown in Fig 1, these options include selecting latitude and longitude of a specific site, selecting site IDs, or dragging a bounding box on the map. Each option requires different inputs as searching criteria. For example, there are two inputs for the latitude/longitude option. One is the latitude and longitude values of a site, which can be chosen directly from the 'Lat/Lon' input widget. The other input is the distance value from a selected site, which can be obtained by dragging the slider of the 'Distance(miles)' widget.

Dashboard pages for all data types except 'Photo' display a well-structured data table for selected sites. For the 'Metadata' dashboard page, it also displays a global map of collected sampling sites. Clicking a marker on the map will return the metadata of the selected site, which includes site ID, location, river name, and sampling date, as shown in Fig. 1. Apart from displaying data tables for a selected site, the 'Hydrograph' dashboard page displays a time-series plot of discharge and/or gage height for the selected site. This allows users to visually assess the quality of the timeseries data and see how gage height and discharge change over time for the site that they are interested in. Dragging the slider of the 'Time range (days)' widget allow users to change the time period of discharge and gage height.

In the 'Photo' dashboard page, four standardized photos of a selected field system are exhibited. This allows users to know the physical environment where the surface water samples were collected. The previously selected subset of data can be exported to a separate folder in the default directory by clicking the 'Save Data' button in the selected dashboard. Users can then use the exported subset of data locally for their research needs.

\section{Availability}

WHONDRS-GUI is available through Shiny Server (https://xmlin.shinyapps.io/whondrs/) for online use, which allows users to get access to the web application through any modern web browser without installing $\mathrm{R}$ or $\mathrm{R}$ studio. The website is free and open to all users without login requirement. The WHONDRS data package as well as the R code are hosted on the ESS-DIVE data archive (https://data.ess-dive.lbl.gov/view/doi:10.15485/1484811). This enables users to simultaneously obtain access to the data and code and to subsequently launch the web app locally from any R environment (e.g., R and RStudio) without any programming or operational system

Peer) reviewing PDF | (2020:02:46163:1:0:CHECK 3 May 2020) 
159

160

161

\section{2}

163

164

165

166

167

168

169

170

171

172

173

174

175

176

177

178

179

180

181

182

183

184

185

186

187

188

189

190

191

192

193

194

195

196

197

198

requirements. Documentation for how to run WHONDRS-GUI locally can be found in the downloaded package.

\section{Results and Discussion}

WHONDRS-GUI, a web application written in R programming language, has been successfully implemented to assist in searching, compiling, visualizing, integrating and exporting different data types of the WHONDRS data package. It makes the ever-growing WHONDRS data package more accessible for researchers to use in performing new analyses or updating prior analyses. As an interactive web application, WHONDRS-GUI is simple and intuitive to use without the need to download and install any specialized software.

The WHONDRS-GUI web application provides interactive data tables, global maps, timeseries plots and standardized river photos of water samples collected across the world. Five data types, which include 'Metadata', 'FTICR', 'Geochemistry', 'Hydrograph' and 'Photo', are being used to demonstrate the collected WHONDRS data. Data processing, subsetting and manipulating were done automatically based on user inputs, such as latitude/ longitude, site ID and distance.

Useful and detailed metadata of the collected samples are available on the 'Metadata' dashboard page. These metadata include site ID, sampling time, location, and river names of the water samples etc. A global map interactively displaying physical locations of selected samples based on user inputs is also available on the same dashboard page (Fig. 1). The global map will automatically zoom in or zoom out according to the number of selected site IDs.

Data tables for all data types except 'Photo' are generated for selected sample sites on corresponding dashboard pages. Fig. 2 shows a preview of the geochemistry data table for seven selected sites in the 'Geochemistry' dashboard page. For the analysis of hydrograph data, depending on the chosen sample ID, a time-series plot of discharge and/or gage height is generated showing the changing pattern of discharge and/or gage height over time, as shown in Fig. 3. The timeseries plot is a variable width plot that defaults to show a time range of 30 days started from the earliest day of the available data. The tine range can be adjusted between 1 day to 365 days using the slider of the 'Time range (days)' widget (Fig. 3).

Apart from the functionality of WHONDRS-GUI demonstrated above, it also provides the option for users who are interested in performing further analyses of the data to download the whole data package or part of it, and to save to local drive, by clicking the 'Save Data' button on the dashboard page. If a user identifies a bug or problem while running the WHONDRS-GUI, it can be reported under the "Issue Report" section. The bug report form is hosted on Google Docs and records each bug report and transmits it to the WHONDRS team automatically.

The results described above show that WHONDRS-GUI provides simple and convenient tools for researchers with limited programming experience to get access to and manipulate the WHONDRS data, and to interactively visualize some of the data types. The web application also offers a solution to integrate large amount of information contained in WHONDRS data with an intuitive and user-friendly interface, which further facilitates the use and exploration of 
199 WHONDRS data by the wider scientific community. However, the current version of the web 200 application focused on the ability to quickly demonstrate available data types, with limited ability 201 to perform data analysis. Also, the format of input and output data used by the web application is 202 limited to CSV file. To improve the flexibility of WHONDRS-GUI and its ability to conduct more

203

204

205

206

207

208

209

210

211

212

213

214

215

216

217

218

219

220

221

222

223

224

225

226

227

228

229

230

231

232

233

234

235

236

237

238 advanced data analysis, we will continue to develop this web application and hopefully to implement these more user-friendly changes in future versions of the web application.

\section{Conclusions}

WHONDRS data are being collected to understand multi-domain connections within river corridors and help predict watershed function. In this work, we developed a web application, WHONDRS-GUI, to make WHONDRS data findable, accessible, interoperable, and reusable (Wilkinson, et al., 2016) and to encourage users to perform their own analyses and extract new information from the data. The WHONDRS-GUI provides a convenient and user-friendly interface specifically for demonstrating different data types of WHONDRS data. It allows users to dynamically interact with the data package. Researchers can utilize WHONDRS-GUI to find the sample data.

The WHONDRS-GUI is an important element of understanding connections among hydrologic, biogeochemical, and microbial function within river corridors. The GUI alone will not provide this understanding, however, and must be coupled with additional analyses and tools as well as additional data types that are not currently in the datasets. For example, detailed molecular properties of dissolved organic matter (DOM) are provided by the FTICR data, which can be paired with data on environmental characteristics (e.g., vegetation type, land use, ecosystem productivity, etc.) that are publicly available. Because all WHONDRS data are georeferenced, these additional environmental data can be extracted from other sources and paired with WHONDRS data to evaluate aspects of the environment that explain variation in DOM chemistry at the global scale. Outcomes of such analyses would provide novel hypotheses to be evaluated through additional sampling, experimentation, and/or modeling.

The data can also be more directly analyzed to understand spatial and temporal variation in biogeochemically-relevant properties, such as the thermodynamic favorability of DOM for microbial oxidation. For example, (Danczak, et al., 2020) recently discovered the principle of 'thermodynamic redundancy,' whereby compositionally distinct metabolomes exhibit consistent levels of thermodynamic favorability. This new principle was revealed, in part, by linking FTICR data to a new conceptual/theoretical framework derived from meta-community ecology (Danczak, et al., 2020). This framework is referred to as 'meta-metabolome ecology' and provides a new window into river corridor function by integrating ecological principles with ultrahigh resolution molecular properties of DOM chemistry. The data necessary to use tools/concepts from metametabolome ecology can be accessed via the WHONDRS-GUI.

To provide additional opportunities to generate new knowledge of integrated river corridor function, WHONDRS is collaborating with the Joint Genome Institute (JGI) to generate metagenomic and metatranscriptomic data of microbial communities. These data can be used with 
239 the FTICR and geochemical data (from the WHONDRS-GUI) to inform biogeochemical reaction 240 network models that can be further integrated with reactive transport codes that simulate reactions 241 and the movement of water and other materials (e.g., nutrients) through river corridors. The 242 develop of such an integrated modeling framework is nascent, and some of the critical theory and 243 modeling tools have recently been developed (Song, et al., 2020). There are significant efforts 244 underway to implement this integrated framework via publicly available tools in the U.S. 245 Department of Energy Systems Biology Knowledgebase (KBase; https://kbase.us/). Once fully 246 implemented, researchers will be able to discover and access WHONDRS data using the GUI and 247 other tools, and then integrate/study these data using tools in KBase that generate and link 248 metabolic, biogeochemical reaction, and reactive transport models. This offers myriad 249 opportunities to generate new knowledge, such as how hydrologic perturbation impacts 250 biogeochemical function via changes to microbial metabolism. Researchers could use numerical 251 in silico experimentation across places and times sampled by WHONDRS to study such impacts 252 and connections. The WHONDRS-GUI is a key piece of the cyberinfrastructure needed to enable 253 such studies and the broader vision of understanding connections among hydrologic, 254 biogeochemical, and microbial function within river corridors.

Both the data package and the $\mathrm{R}$ code are hosted on the ESS-DIVE data archive (https://data.ess-dive.lbl.gov/view/doi:10.15485/1484811), which enables users to simultaneously obtain access to the data and code and to subsequently run the web app locally. The WHONDRSGUI is also available for online use at Shiny Server (https://xmlin.shinyapps.io/whondrs/).

\section{Acknowledgements}

We would like to thank all collaborators for collecting the surface water samples, and the EMSL 263 teams for conducting a series of metabolomics analysis and geochemical analyses.

\section{References}

Chang, W. and Borges Ribeiro, B.J.R.p.v. Shinydashboard: create dashboards with'Shiny'. $2017 ; 1$.

Danczak, R.E., et al. Unification of environmental metabolomics with metacommunity ecology. bioRxiv 2020.

Danczak, R.E., et al. Ecological theory applied to environmental metabolomes reveals compositional divergence despite conserved molecular properties. bioRxiv 2020.

Graham, E.B., et al. Subsurface biogeochemistry is a missing link between ecology and hydrology in dam-impacted river corridors. Science of the Total Environment 2019;657:435-445.

Harvey, J. and Gooseff, M. River corridor science: Hydrologic exchange and ecological consequences from bedforms to basins. Water Resources Research 2015;51(9):6893-6922.

Marshall, A.G., Hendrickson, C.L. and Jackson, G.S.J.M.s.r. Fourier transform ion cyclotron resonance mass spectrometry: a primer. 1998;17(1):1-35.

Postel, S.L. and Thompson Jr, B.H. Watershed protection: Capturing the benefits of nature's water supply services. In, Natural Resources Forum. Wiley Online Library; 2005. p. 98-108. 
279 R.Core.Team. R: A Language and Environment for Statistical Computing. 2018.

280 Scheibe, T., et al. Data-Model Integration for Improved Prediction of River Corridor and 281 Watershed Function. In, AGU Fall Meeting Abstracts. 2018.

282 Song, H.-S., et al. Representing Organic Matter Thermodynamics in Biogeochemical Reactions 283 via Substrate-Explicit Modeling. bioRxiv 2020.

284 Stegen, J.C. and Goldman, A.E. WHONDRS: a Community Resource for Studying Dynamic River 285 Corridors. In.: Am Soc Microbiol; 2018.

286 Stegen, J.C., et al. WHONDRS Surface Water Sampling for Metabolite Biogeography. In.: 287 Environmental System Science Data Infrastructure for a Virtual Ecosystem ...; 2018.

288 Wickham, H., et al. dplyr: A grammar of data manipulation. R package version 0.4 2015;3.

289 Wilkinson, M.D., et al. The FAIR Guiding Principles for scientific data management and 290 stewardship. Scientific data 2016;3.

291 Winston Chang, J.C., JJ Allaire, Yihui Xie and Jonathan McPherson. shiny: Web Application $292 \quad$ Framework for R. 2018.

293 


\section{Figure 1}

Figure 1. Metadata dashboard page.

The 'Metadata' dashboard page shows a global map of collected sampling sites. User input widgets on the top of the page, including 'Latitude/Longtitude', 'Bounding Box', 'Site ID', or 'All Stie IDs', allow users to search for a subset of data based on different options. Clicking a marker on the map will return the metadata of the selected site, as shown in the map.

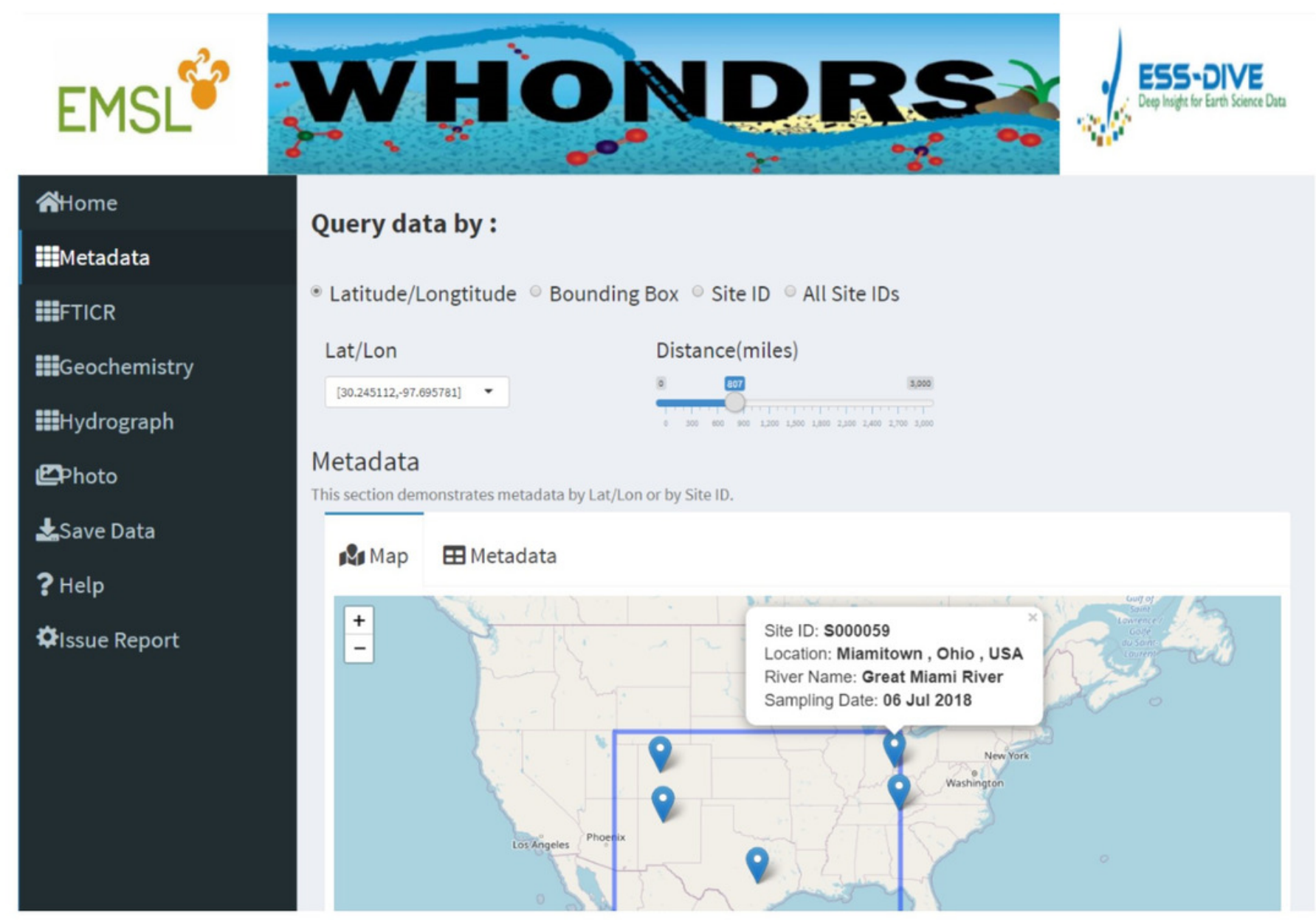


Figure 2

Figure 2.Geochemistry dashboard page.

A preview of the geochemistry data table for seven selected sites.

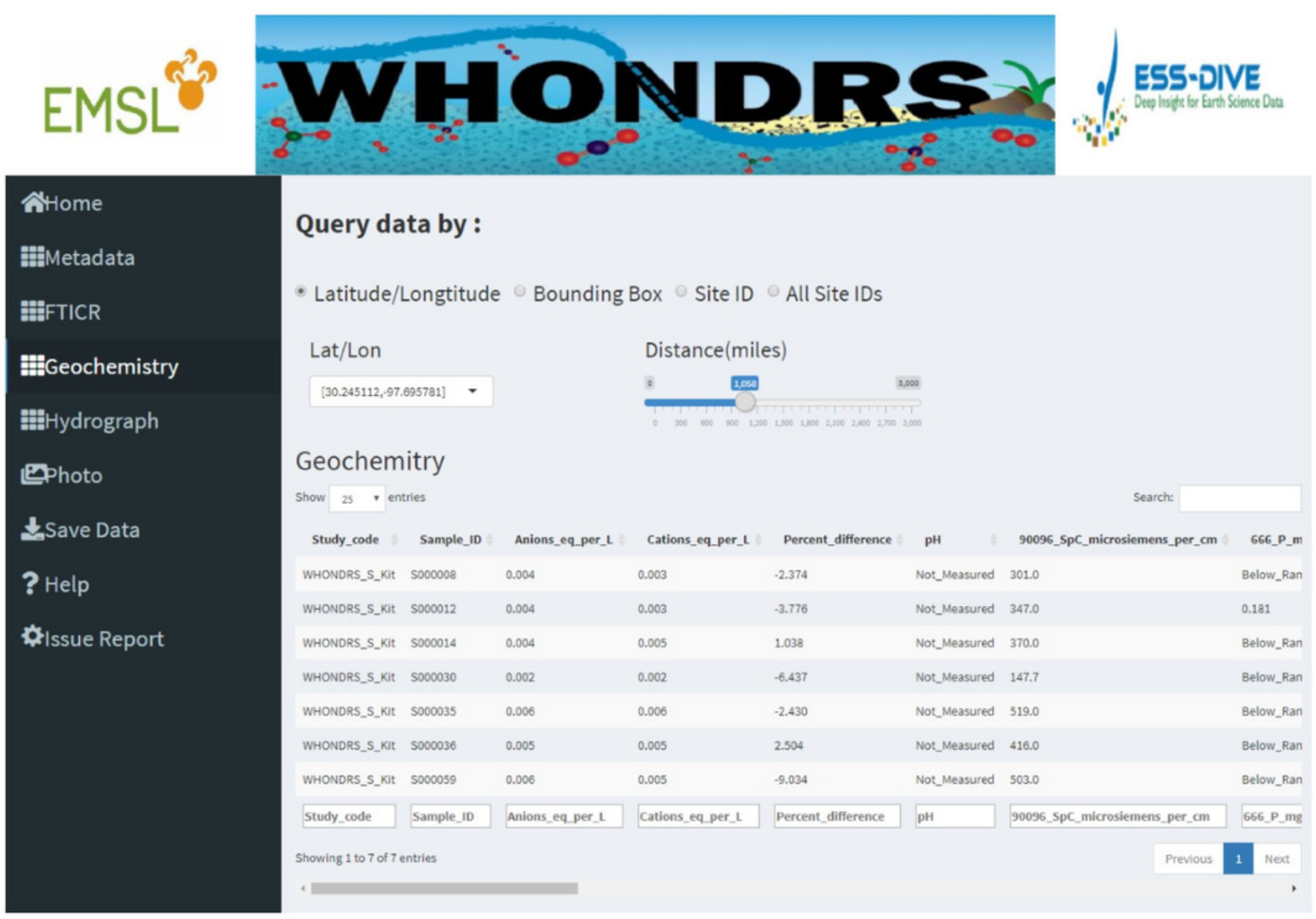




\section{Figure 3}

Figure 3. Hydrograph dashboard page.

This dashboard page displays an interactive time-series plot of discharge and/or gage height for a selected site. The plot shown in this fugure is a time-series plot of discharge and gage height for site 'S000009'.

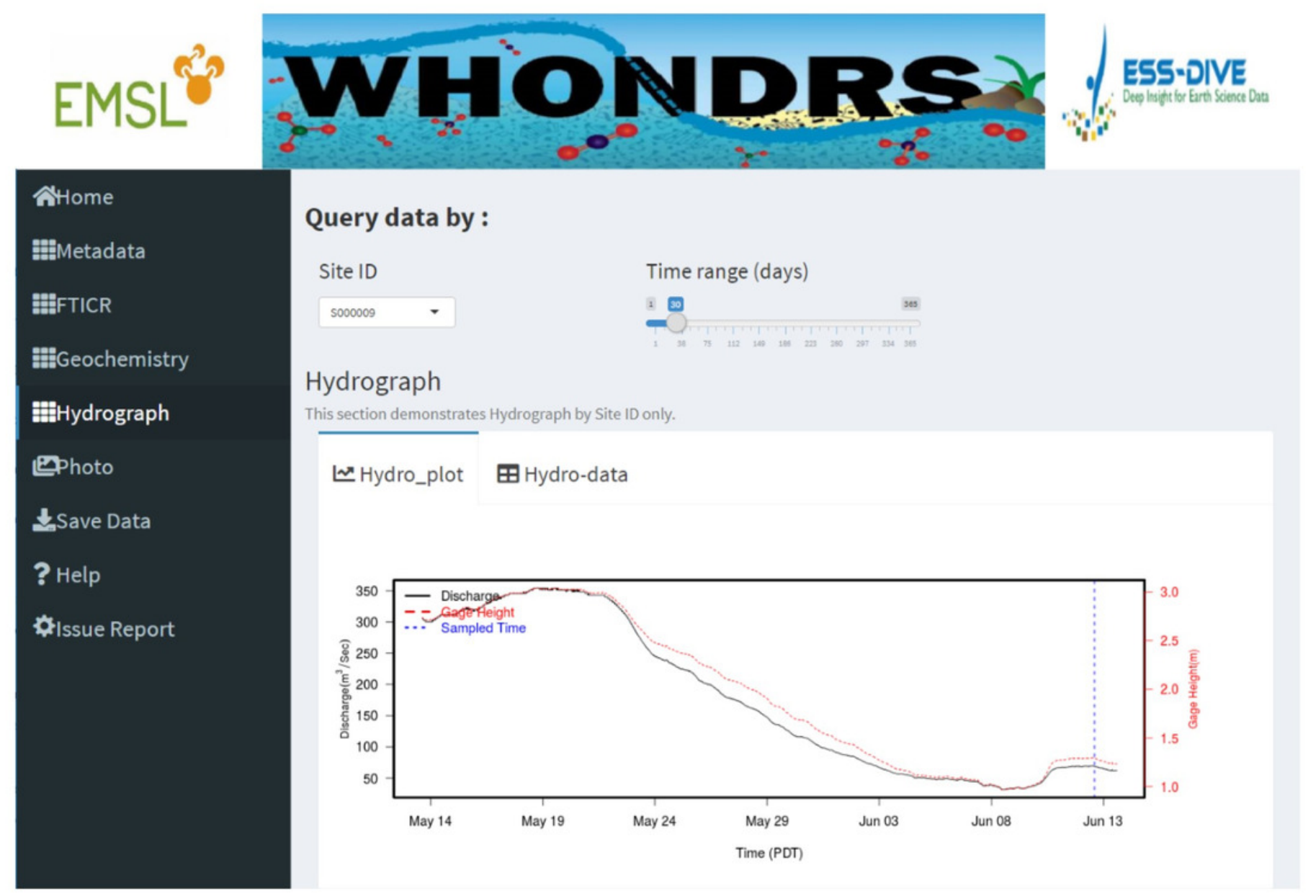

